\title{
Supermodes of Grating-Coupled Multimode Waveguides and Application to Mode Conversion Between Copropagating Modes Mediated by Backward Bragg Scattering
}

\author{
Eva Peral and Amnon Yariv, Life Fellow, IEEE
}

\begin{abstract}
An analysis of multimode waveguides where several modes are coupled via quasiperiodic perturbations is presented. The supermodes (or eigenmodes) of the structure are derived and orthonormality considerations are discussed. In addition, a new type of mode converter between copropagating modes is proposed, where mode conversion is mediated by a backwardpropagating mode. Adiabatic and nonadiabatic coupling coefficients are considered and the supermode formalism is used to conveniently describe the mode of operation of the device.
\end{abstract}

Index Terms - Coupled mode analysis, gratings, multimode waveguides, optical fiber communications, optical fiber devices, optical fiber theory.

\section{INTRODUCTION}

$\mathbf{T}$ HE theory of coupled modes for guided wave optics [1] was developed for treating interactions between, mostly, confined modes of optical waveguides which are mediated by refractive index perturbation. Two of the most important examples are those of periodic index perturbation (distributed feedback (DFB) lasers [2] and Bragg fiber gratings [3]) and coupling via evanescent fields in parallel waveguide systems [4].

As a result of the perturbation, the modes of the structure are modified, and the propagating eigenmodes, the so-called supermodes, of the perturbed structure [4], [5] have to be found. For quasiperiodic perturbations, these are the Floquet-Bloch modes [6]-[8].

The use of Floquet-Bloch modes becomes a practical necessity in analyzing a new and emerging class of wavelength division multiplexed (WDM)-related devices [9] based on adiabatic transitions and/or where several waveguide modes might be involved.

Here we will derive formal expressions for the supermodes of systems with an arbitrary number of modes. The supermodes, in these cases, become the natural new building blocks in which to analyze phenomena and devices. A metric is

Manuscript received August 14, 1998; revised February 5, 1999. This work was supported by the Defense Advanced Research Projects Agency the Office of Naval Research and the Air Force Office of Scientific Research under Award N00014-91-J-1195.

The authors are with the Department of Applied Physics, California Institute of Technology, Pasadena, CA 91125 USA (e-mail: eva@ cco.caltech.edu).

Publisher Item Identifier S 0733-8724(99)03808-6. derived from considerations of power flux conservation to ensure orthonormality of the supermodes. The usefulness of this approach will be demonstrated in the analysis of a new type of mode converter between copropagating modes mediated by a backward propagating mode. Adiabatic transitions will be considered.

\section{Generalized Coupled Mode Theory}

The transverse modes of a dielectric waveguide, which form a complete orthonormal set, cease to be proper modes when a spatial modulation of the index of refraction or the gain (loss) is present, and the new eigenmodes, which we call supermodes, have to be determined.

The original modes of the unperturbed waveguide are used as an orthonormal basis set. Because of conservation of photon energy and momentum, a quasiperiodic perturbation couples usually a small number of modes, $N$. The electric field of the new mode is taken as a superposition of the orthonormal unperturbed waveguide modes [4], [10]

$$
\vec{E}(x, y, z, t)=\sum_{i} A_{i}(z) \vec{E}_{i}(x, y) e^{-i \beta_{i} z} e^{i \omega t}
$$

with $\beta_{i}>0$ for forward propagating modes, and $\beta_{i}<0$ for modes propagating in the $-z$ direction.

The orthonormality condition is expressed, in the transverse mode approximation, as [10]

$$
\iint_{-\infty}^{\infty} \vec{E}_{i}(x, y) \cdot \vec{E}_{j}^{*}(x, y) d x d y=\frac{2 \omega \mu}{\left|\beta_{i}\right|} \delta_{i j}
$$

so that the power carried by the mode $i$ is $\left|A_{i}\right|^{2}$. In an unperturbed waveguide the complex mode amplitudes $A_{i}$ are arbitrary constants that are determined by the boundary conditions.

The mode amplitudes $A_{i}$ are, in general, $z$ dependent and obey the coupled mode equations [1]

$$
\begin{aligned}
\frac{d A_{i}}{d z} & =\sum_{j \neq i}-i \widetilde{\kappa}_{i j} \exp \left[-i\left(\beta_{j}-\beta_{i}+\frac{2 \pi}{\Lambda_{i j}}\right) z\right] A_{j} \\
& =\sum_{j \neq i}-i \widetilde{\kappa}_{i j} \exp \left[i 2 \delta_{i j} z\right] A_{j}
\end{aligned}
$$


where $A_{i}$ are the mode amplitudes, $\beta_{i}$ the propagation constants and $\tilde{\kappa}_{i j}$ and $\Lambda_{i j}$ the amplitude and period, respectively, of the Bragg grating that couples modes $i$ and $j$. The detuning $\delta_{i j}$ is defined as

$$
2 \delta_{i j}=\beta_{i}-\beta_{j}-\frac{2 \pi}{\Lambda_{i j}} .
$$

The sign of the Bragg period $\Lambda_{i j}$ is taken so that near the filter center frequency $\omega_{0}$ the detuning is small, $\delta_{i j} \approx 0$, i.e., the modes are strongly coupled. Thus, $\delta_{i j}=-\delta_{j i}$. If the modes $i$ and $j$ propagate in the same direction, then $\tilde{\kappa}_{i j}=\tilde{\kappa}_{j i}^{*}$, otherwise, if the modes propagate in opposite directions, $\tilde{\kappa}_{i j}=-\tilde{\kappa}_{j i}^{*}$.

It is convenient to remove the rapidly varying oscillatory term in the coupled mode equations (3) and use new mode amplitudes defined as

$$
a_{i}=A_{i} \exp \left(-i \delta_{i} z\right)
$$

where the reference detunings $\delta_{i}$ have yet to be determined. On substitution in the coupled mode equations (3), the new equations are given by

$$
\frac{d a_{i}}{d z}=-i \delta_{i} a_{i}-i \sum_{j \neq i} \tilde{\kappa}_{i j} \exp \left[i\left(2 \delta_{i j}-\delta_{i}+\delta_{j}\right) z\right] a_{j}
$$

By defining new coupling coefficients

$$
\kappa_{i j}=\tilde{\kappa}_{i j} \exp \left[i\left(2 \delta_{i j}-\delta_{i}+\delta_{j}\right) z\right]
$$

the coupled mode equations become

$$
\frac{d a_{i}}{d z}=-i \delta_{i} a_{i}-i \sum_{j \neq i} \kappa_{i j} a_{j}
$$

If the number of coupling coefficients, $\tilde{\kappa}_{i j}$, different from zero is less than or equal to the number of modes, $N$, which is always satisfied for bimodal or trimodal structures, the reference detunings, $\delta_{i}$, can be chosen so that

$$
2 \delta_{i j}-\delta_{i}+\delta_{j}=0
$$

and as a consequence, $\kappa_{i j}=\tilde{\kappa}_{i j}$. Otherwise, the new coupling coefficients $\kappa_{i j}$ have a phase, which depends linearly on $z$.

The coupled wave equations can be expressed in matrix form as

$$
i \frac{d a}{d z}=\left[\begin{array}{cccc}
\delta_{1} & \kappa_{12} & \cdots & \kappa_{1 N} \\
\kappa_{21} & \delta_{2} & & \\
\vdots & & \ddots & \vdots \\
\kappa_{N 1} & & \cdots & \delta_{N}
\end{array}\right] a=H a
$$

where $a=\left[\begin{array}{llll}a_{1} & a_{2} & \cdots & a_{N}\end{array}\right]^{T}$. If the coupling coefficients $\kappa_{i j}$ do not depend on $z$, or vary very slowly (as in an adiabatic transition), we can postulate that the field components evolve as

$$
a(z)=a(0) \exp (-i \gamma z)
$$

which when used in (10) leads to

$$
i \frac{d a}{d z}=\gamma a=H a \text {. }
$$

This is an eigenvalue equation that yields the (adiabatic) eigenvectors or supermodes and eigenvalues of the structure.

\section{ON SUPERMODE ORTHONORMALITY AND POWER CONSERVATION}

In the previous section, the supermodes of a grating-coupled multimode waveguide were derived. However, in order that any field can be easily expanded as a superposition of these modes, it is convenient that these supermodes are also orthonormal among themselves. However, if there are counterpropagating fields, under the commonly employed unitary inner product the supermodes are not orthogonal and power flux conservation is not obtained. In this section a new metric is derived from considerations of conservation of power flux that yields an orthonormality condition for both co- and contradirectional coupling.

We consider the general case of $N_{1}$ modes propagating in the $+z$ direction and $N_{2}$ modes traveling in the $-z$ direction. The amplitudes $a_{i}$ of these modes form the vectors $R$ and $S$, respectively. Thus, we can define the vector $a=\left[\begin{array}{c}R \\ S\end{array}\right]$ and the matrix system (10) is then given by

$$
i \frac{d}{d z}\left[\begin{array}{l}
R \\
S
\end{array}\right]=H\left[\begin{array}{l}
R \\
S
\end{array}\right]=\left[\begin{array}{cc}
\kappa_{R R} & \kappa_{R S} \\
-\kappa_{R S}^{\dagger} & \kappa_{S S}
\end{array}\right]\left[\begin{array}{l}
R \\
S
\end{array}\right]
$$

where the superscript $\dagger$ indicates hermitian conjugate. $\kappa_{R R}$ and $\kappa_{S S}$ are Hermitian matrices with dimensions $N_{1} \times N_{1}$ and $N_{2} \times N_{2}$, respectively.

When modes travel in different directions, the total power flow is given by

$$
P=a^{\dagger} K a
$$

where $\left(a^{+}\right)_{i j} \equiv a_{j i}^{*}$ and

$$
K=\left[\begin{array}{cc}
I_{N_{1}} & 0 \\
0 & -I_{N_{2}}
\end{array}\right] \text {. }
$$

Here $I_{N_{i}}$ is the identity matrix of dimension $N_{i} \times N_{i}$. Thus, $K$ is a parity matrix consisting of \pm 1 in the main diagonal. Equation (14) can be expressed as

$$
P=\langle a, a\rangle
$$

where $\langle a, b\rangle$ is an inner product defined as

$$
\langle a, b\rangle=a^{\dagger} K b
$$

and $K$ is the metric. With the matrix $K$ defined in (15), this is an improper product, i.e., $K$ is indefinite, and the inner product can be a negative number. This would correspond physically to power flowing in the direction of negative $z$.

The power flux is a conserved quantity, i.e., independent of $z$. This requirement imposes a condition on the system matrix $H$. Using (10) and (14), we get

$$
\frac{d P}{d z}=\frac{d a^{\dagger}}{d z} K a+a^{\dagger} K \frac{d a}{d z}=i a^{\dagger}\left(H^{\dagger} K-H K\right) a=0 .
$$

Thus, $H$ has to satisfy

$$
H=K^{-1} H^{\dagger} K \equiv H^{\#} .
$$

In the equation above, $H^{\#}$ is the adjoint of $H$ in this metric, and the $H$ satisfying $H=H^{\#}$ are referred to as $K$-Hermitian 


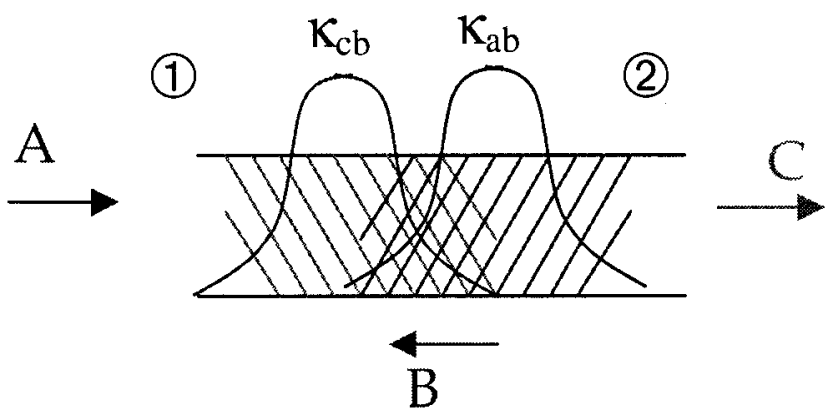

Fig. 1. Mode converter mediated by backward Bragg scattering.

matrices. By direct substitution, it can be shown that the $H$ in (13) with the metric in (15) is $K$-Hermitian.

It can be shown [13] that the eigenvalues, $\gamma_{i}$, of $K$ hermitian matrices are either real or else occur in complex conjugate pairs. If we denote $\gamma_{i}$ the eigenvalue associated with the eigenvector $x_{i}$, then $x_{i}$ is $K$-orthogonal to all the other eigenvectors, $x_{j}$, with eigenvalue $\gamma_{j}$ different from $\gamma_{i}^{*}$, that is

$$
\begin{array}{ll}
\left\langle x_{i}, x_{j}\right\rangle=0 & \text { for all } j \text { such that } \gamma_{j} \neq \gamma_{i}^{*} \\
\left\langle x_{i}, x_{j}\right\rangle \neq 0 & \text { for } j \text { such that } \gamma_{j}=\gamma_{i}^{*} .
\end{array}
$$

Thus, in the case of a complex eigenvalue, orthogonality is defined in a different way. The eigenvector associated with the complex eigenvalue is orthogonal to itself but is not orthogonal to the eigenvector associated with the complex conjugate eigenvalue.

If the eigenvalues are not degenerate, then the eigenvectors form a complete set and any vector can be expressed as a linear combination of them. The coefficients of the linear expansion are obtained using (20).

As an application of these concepts, mode conversion between two copropagating modes mediated by a backward propagating mode is analyzed in the following section.

\section{Mode Conversion Between Copropagating Modes}

A mode converter is a device that converts light propagating in one mode of a waveguide to another mode. Mode conversion has been used in various applications such as optical sensors, rocking filters, and dispersion compensators. In previous works, mode conversion was achieved by coupling of copropagating modes using long-period gratings [14]. This has several drawbacks such as long devices and it is necessary to precisely control the length of the coupling region in order to achieve maximum coupling efficiency. This is, to our knowledge, the first time that a mode converter is proposed that is based on coupling of counterpropagating mode, and yet input and output modes travel in the same direction.

The structure of the mode converter is shown in Fig. 1. The device should be fabricated in a waveguide that supports three propagating modes. Coupling between two forward propagating modes, $\mathrm{A}$ and $\mathrm{C}$, and a backward propagating mode, $\mathrm{B}$ occur due to two gratings, the first one couples, i.e., transfers power, between oppositely traveling modes $\mathrm{B}$ and $\mathrm{C}$, whereas the second one couples oppositely traveling modes B and A. Modes $\mathrm{A}$ and $\mathrm{C}$ are not coupled directly by any of the gratings.
The principle of operation is as follows. When mode A is incident on port 1, it travels forward unaffected by the first grating. When mode A reaches the second grating, it is reflected back into mode B. Mode B then travels backward and reaches the first grating, where it is reflected back into mode C. Mode $\mathrm{C}$ travels forward along the second grating, which has no effect on it. As a result mode $\mathrm{C}$ emerges from port 2. In addition, if mode $\mathrm{C}$ were incident on port 1 , it would be very strongly reflected.

The system matrix associated with this device is given by

$$
H=\left[\begin{array}{ccc}
0 & \kappa_{a b} & 0 \\
-\kappa_{a b}^{*} & \delta_{b} & -\kappa_{c b}^{*} \\
0 & \kappa_{c b} & \delta_{c}
\end{array}\right]
$$

Here, we have set arbitrarily the reference detuning $\delta_{a}$ to zero, so that from (9)

$$
\begin{aligned}
& \delta_{b}=-2 \delta_{a b} \\
& \delta_{c}=-2\left(\delta_{a b}-\delta_{c b}\right) .
\end{aligned}
$$

In order to find the eigenvalues of $H$, the following characteristic equation has to be solved

$\gamma^{3}-\gamma^{2}\left(\delta_{b}+\delta_{c}\right)+\gamma\left(\delta_{b} \delta_{c}+\left|\kappa_{a b}\right|^{2}+\left|\kappa_{c b}\right|^{2}\right)-\delta_{c}\left|\kappa_{a b}\right|^{2}=0$

One of these eigenvalues, $\gamma_{0}$, is always real, whereas the other two, $\gamma_{ \pm}$, can be complex conjugate, inside the so-called forbidden region, or both real, outside of the forbidden region. On the boundary the two supermodes $x_{ \pm}$are degenerate.

The eigenvectors are given by

$$
\begin{gathered}
x_{i}=\left[\begin{array}{ccc}
\kappa_{a b} & \gamma_{i} & \frac{-\left|\kappa_{a b}\right|^{2}+\left(\delta_{b}-\gamma_{i}\right) \gamma_{i}}{\kappa_{c b}^{*}}
\end{array}\right]^{T} \\
\text { where } i=0,+,-.
\end{gathered}
$$

In consideration of the discussion in the previous section, inside the forbidden region the eigenvectors $x_{0}$ and $x_{ \pm}$can be normalized so as to satisfy the following orthonormality relations

$$
\begin{aligned}
\left\langle x_{0}, x_{ \pm}\right\rangle=0, & \left\langle x_{0}, x_{0}\right\rangle=1 \\
\left\langle x_{ \pm}, x_{ \pm}\right\rangle=0, & \left\langle x_{ \pm}, x_{\mp}\right\rangle=1
\end{aligned}
$$

whereas outside of the forbidden region

$$
\begin{aligned}
\left\langle x_{0}, x_{ \pm}\right\rangle=0, & \left\langle x_{0}, x_{0}\right\rangle=1 \\
\left\langle x_{ \pm}, x_{\mp}\right\rangle=0, \quad & \left\langle x_{ \pm}, x_{ \pm}\right\rangle=1 .
\end{aligned}
$$

On the boundary, the eigenvalues are degenerate, and the eigenvectors do not form a complete set.

Although an analytic solution exists in the general case (see Appendix A), it is very involved and difficult to interpret. Here we will limit ourselves to the case of $\delta_{c}=-2\left(\delta_{a b}-\delta_{c b}\right)=0$, which will be denoted as two-photon resonance. In this case, the eigenvalues are given by:

$$
\gamma_{0}=0 ; \quad \gamma_{ \pm}=\frac{\delta_{b}}{2} \pm i \sqrt{\left(\left|\kappa_{a b}\right|^{2}+\left|\kappa_{c b}\right|^{2}\right)-\left(\frac{\delta_{b}}{2}\right)^{2}}
$$


We further confine ourselves to the region inside the forbidden gap, that is

$$
\left|\kappa_{a b}\right|^{2}+\left|\kappa_{c b}\right|^{2}>\left(\frac{\delta_{b}}{2}\right)^{2}
$$

and assume that the coupling coefficients, $\kappa_{a b}$ and $\kappa_{c b}$ are real and positive. Normalized parameters can be defined

$$
r=\frac{\kappa_{c b}}{\kappa_{a b}} ; \quad \Delta=\frac{\delta_{b}}{2 \kappa_{a b}}
$$

so that the eigenvalues take the form

$$
\gamma_{0}=0 ; \quad \gamma_{ \pm}=\kappa_{a b}\left(\Delta \pm i \sqrt{1+r^{2}-\Delta^{2}}\right)
$$

and the corresponding normalized eigenvectors satisfying (25) are

$$
\begin{aligned}
x_{0} & =\frac{1}{\sqrt{1+r^{2}}}\left[\begin{array}{lll}
r & 0 & -1
\end{array}\right]^{T} \\
x_{ \pm} & =\frac{e^{\mp i(\alpha / 2)}}{\sqrt{2 \cos \alpha} \sqrt{1+r^{2}}}\left[\begin{array}{lll}
1 & \mp i \sqrt{1+r^{2}} e^{ \pm i \alpha} & r
\end{array}\right]^{T}
\end{aligned}
$$

where

$$
\sin \alpha=-\frac{\Delta}{\sqrt{1+r^{2}}}, \quad \alpha \in\left[-\frac{\pi}{2}, \frac{\pi}{2}\right] .
$$

We define a "canonical basis" in terms of the uncoupled waveguide modes

$$
e_{a}=\left[\begin{array}{l}
1 \\
0 \\
0
\end{array}\right], \quad e_{b}=\left[\begin{array}{l}
0 \\
1 \\
0
\end{array}\right], \quad e_{c}=\left[\begin{array}{l}
0 \\
0 \\
1
\end{array}\right] .
$$

These three vectors are orthogonal and normalized to unity power flow, and correspond to the unperturbed waveguide modes A, B, and C. This is an unusual basis in the sense that the power flow associated with the second mode, $e_{b}$, is negative, whereas modes $e_{a}$ and $e_{c}$, carry positive power flow. The eigenvectors can be expressed as a combination of these canonical modes

$$
\begin{aligned}
& x_{0}=\frac{1}{\sqrt{1+r^{2}}}\left(r e_{a}-e_{c}\right) \\
& x_{ \pm}=\frac{e^{\mp i(\alpha / 2)}}{\sqrt{2 \cos \alpha} \sqrt{1+r^{2}}}\left(e_{a} \mp i \sqrt{1+r^{2}} e^{ \pm i \alpha} e_{b}+r e_{c}\right) .
\end{aligned}
$$

The analysis above enables us to predict the behavior of the device in the adiabatic regime. At the input, and according to Fig. 1, $r$ tends to infinity, and if the canonical mode $e_{a}$ is incident in the mode converter, then the supermode $x_{0}$ will be excited. The supermode $x_{0}$ corresponds to eigenvalue zero, so in the adiabatic approximation, if excited initially, it will propagate along the structure unperturbed. At the output, $r$ tends to zero, and according to the expression of the supermode $x_{0}$ in (34), the canonical mode $e_{c}$ will exit the device. Thus, we have achieved mode conversion from mode $e_{a}$ to mode $e_{c}$ with ideally no energy transfer to mode $e_{b}$. The validity of the adiabaticity condition is discussed in Appendix B.

The validity of this analysis has been tested by solving the coupled wave equations (3) numerically. In Fig. 2, the mode amplitudes have been plotted as a function of device length for

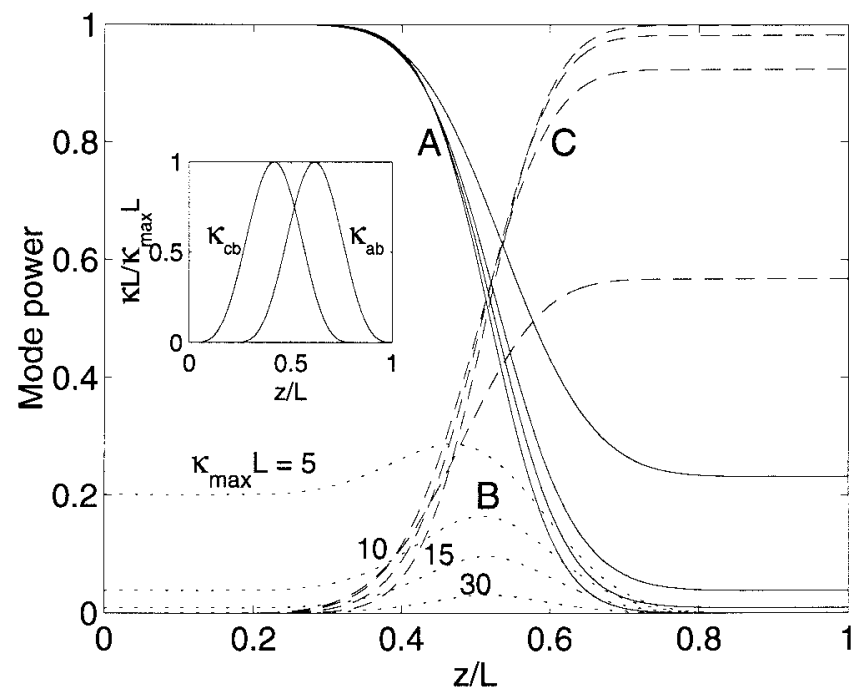

Fig. 2. Mode power for quasiadiabatic mode converter on resonance for several coupling strengths. The inset shows the coupling coefficients.

quasiadiabatic Gaussian-shaped coupling coefficients (shown in the inset) with several strengths. As expected, the backward mode, B, is hardly excited. As the magnitude of the coupling coefficient increases, the adiabaticity condition is more valid (as explained in Appendix B), and the energy transfer to this mode decreases substantially.

In the case of uniform coupling coefficients, an analytic solution can be obtained for the propagating field by using the supermodes previously derived. If the two gratings do not overlap completely, three regions are distinct, and the field is a superposition of the supermodes, where the coefficients are found by applying the boundary conditions. In Appendix A, we derive the equation for the boundary of the forbidden region, inside which some eigenvalues are complex. Inside the forbidden region, the reflection coefficient is expected to be uniform, whereas outside it, all the eigenvalues are real, and the reflection has an oscillatory behavior. This is shown in the contour plot in Fig. 3, where the reflection coefficient at the input of a device with two overlapping uniform gratings is plotted and as a function of the frequency deviations, $\delta_{a b}$ and $\delta_{b c}$. The boundary of the forbidden region is shown with a thick solid line. Near resonance for both gratings, the reflection coefficient decreases because part of the input mode $\mathrm{A}$ is converted into mode $\mathrm{C}$. When the first grating that couples $\mathrm{B}$ and $\mathrm{C}$ is off resonance, there is a large reflection due to the second grating. When both gratings are off resonance, outside the forbidden region, the reflectivity decreases in an oscillatory fashion.

\section{CONCLUSION}

An analysis of multimode waveguides where the modes are coupled through quasiperiodic perturbations has been presented, which yielded the supermodes of the structure. An orthonormality condition has been derived using conservation of power flux, which is valid for both co- and contradirectional modes. As an example, a mode converter of forward propagating modes has been proposed where conversion from 


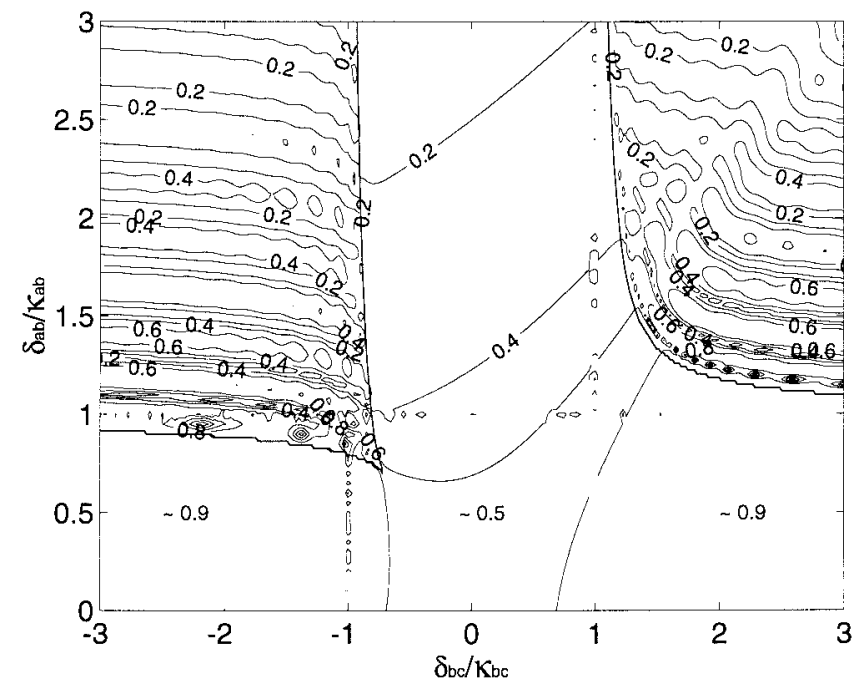

Fig. 3. Contour plot of the reflection coefficient at the input of a uniform mode converter with $\kappa_{c b} L=\kappa_{a b} L=20$. The thick solid line is the boundary of the forbidden region.

one forward mode to another is mediated by a backward propagating mode. In the adiabatic regime, this backward mode, although required, is never excited. An analysis of the structure with adiabatic coupling coefficients has proved the usefulness of the supermode formalism for analyzing complex structures.

\section{APPENDIX A}

GENERAL SOLUTION FOR THE EIGENVALUES OF THE MODE CONVERTER

The characteristic equation (23) is a cubic equation that has an analytical solution [15]. If we define the parameters

$$
\begin{aligned}
p= & \frac{1}{3}\left(\delta_{b} \delta_{c}+\left|\kappa_{a b}\right|^{2}+\left|\kappa_{c b}\right|^{2}\right)-\frac{1}{9}\left(\delta_{b}+\delta_{c}\right)^{2} \\
q= & -\frac{1}{27}\left(\delta_{b}+\delta_{c}\right)^{3}+\frac{1}{6}\left(\delta_{b}+\delta_{c}\right)\left(\delta_{b} \delta_{c}+\left|\kappa_{a b}\right|^{2}+\left|\kappa_{c b}\right|^{2}\right) \\
& -\frac{1}{2} \delta_{c}\left|\kappa_{a b}\right|^{2}
\end{aligned}
$$

then the discriminant is given by $q^{2}+p^{3}$. If the discriminant is positive, then one root is real and two are complex conjugates. If the discriminant is zero, then there are three real roots, of which at least two are equal. If the discriminant is negative, then there are three unequal real roots. Thus, the equation $q^{2}+p^{3}=0$ describes the boundary of the forbidden region. The eigenvalues are given by

$$
\begin{aligned}
& \gamma_{0}=\frac{1}{3}\left(\delta_{b}+\delta_{c}\right)+\lambda_{+} \\
& \gamma_{ \pm}=\frac{1}{3}\left(\delta_{b}+\delta_{c}\right)-\frac{1}{2} \lambda_{+} \pm i \frac{\sqrt{3}}{2} \lambda_{-}
\end{aligned}
$$

where

$$
\lambda_{ \pm}=\left(-q+\sqrt{q^{2}+p^{3}}\right)^{1 / 3} \pm\left(-q-\sqrt{q^{2}+p^{3}}\right)^{1 / 3} .
$$

The eigenvalue $\gamma_{0}$ is always real whereas $\gamma_{ \pm}$are complex conjugate inside the forbidden region, i.e., for $q^{2}+p^{3}>0$, are real outside of it, and are equal at the boundary.

\section{APPENDIX B}

\section{VALIDITY OF THE ADIABATICITY CONDITION}

In the following, the adibaticity condition is discussed. The system matrix (21) can be transformed from the canonical basis to the basis composed by the adiabatic supermodes. Defining the matrix $X$ as

$$
X=\left[\begin{array}{ccc}
\mid & \mid & \mid \\
x_{+} & x_{0} & x_{-} \\
\mid & \mid & \mid
\end{array}\right]
$$

the amplitudes of the eigenmodes, $a_{0}$ and $a_{ \pm}$, satisfy the equation

$$
i \frac{d}{d z}\left[\begin{array}{c}
a_{+} \\
a_{0} \\
a_{-}
\end{array}\right]=H_{a d}\left[\begin{array}{c}
a_{+} \\
a_{0} \\
a_{-}
\end{array}\right]
$$

where the adiabatic system matrix, $H_{a d}$, is given by

$$
H_{a d}=X^{-1} H X-i X^{-1} \frac{d}{d z} X .
$$

The matrix $X$ can be expressed in a more convenient way by defining the angles $\theta$ and $\varphi$ such that

$$
\begin{aligned}
\sin \theta=\frac{1}{\sqrt{1+r^{2}}}, \quad \cos \theta=\frac{r}{\sqrt{1+r^{2}}} \\
\sin \varphi=\frac{e^{-i(\alpha / 2)}}{\sqrt{2 \cos \alpha}}, \quad \cos \varphi=\frac{e^{i(\alpha / 2)}}{\sqrt{2 \cos \alpha}} .
\end{aligned}
$$

Thus

$$
X=\left[\begin{array}{ccc}
\sin \theta \sin \varphi & \cos \theta & \sin \theta \cos \varphi \\
-i \cos \varphi & 0 & i \sin \varphi \\
\cos \theta \sin \varphi & -\sin \theta & \cos \theta \cos \varphi
\end{array}\right] .
$$

This is similar to a rotation with angles $\theta$ and $\varphi$. However, in this case $\varphi$ is a complex angle, and the transformation is not $K$-unitary because, as mentioned above, the canonical modes are not all normalized to the same value.

Using the orthonormality relations (25), it can be shown that

$$
X^{\dagger} K X=\tilde{I}
$$

where

$$
\tilde{I}=\left[\begin{array}{lll}
0 & 0 & 1 \\
0 & 1 & 0 \\
1 & 0 & 0
\end{array}\right]
$$

Thus

$$
X^{-1}=\tilde{I} X^{\dagger} K
$$

and substituting $X$ from (42) we get

$$
X^{-1}=\left[\begin{array}{ccc}
\sin \theta \sin \varphi & i \cos \varphi & \cos \theta \sin \varphi \\
\cos \theta & 0 & -\sin \theta \\
\sin \theta \cos \varphi & -i \sin \varphi & \cos \theta \cos \varphi
\end{array}\right] .
$$

Then, substituting $X$ and $X^{-1}$ in (40), we obtain (47) shown at the top of the next page, where an overdot indicates the derivative with respect to $z$. 


$$
H_{a d}=i\left[\begin{array}{ccc}
\kappa_{a b} \frac{-\sin 2 \varphi+\cos 2 \varphi \cot \varphi}{\sin \theta} & \dot{\theta} \sin \varphi & \dot{\varphi}-\kappa_{a b} \frac{\cos 2 \varphi}{\sin \theta} \\
-\dot{\theta} \sin \varphi & 0 & -\dot{\theta} \cos \varphi \\
-\dot{\varphi}-\kappa_{a b} \frac{\cos 2 \varphi}{\sin \theta} & \dot{\theta} \cos \varphi & \kappa_{a b} \frac{\sin 2 \varphi+\cos 2 \varphi c \tan \varphi}{\sin \theta}
\end{array}\right]
$$

On resonance the matrix above simplifies to

$$
H_{a d}=\frac{i}{\sqrt{2}}\left[\begin{array}{ccc}
-\sqrt{2} \kappa_{a b} \operatorname{cosec} \theta & \dot{\theta} & 0 \\
-\dot{\theta} & 0 & -\dot{\theta} \\
0 & \dot{\theta} & \sqrt{2} \kappa_{a b} \operatorname{cosec} \theta
\end{array}\right] .
$$

Adiabatic evolution takes place if the adiabatic supermode $x_{0}$ is effectively decoupled from the other two adiabatic supermodes $x_{+}$and $x_{-}$. According to (47), this will be the case if the nondiagonal elements involving $\dot{\theta}$ are small compared to the nonzero terms in the diagonal. On resonance the adiabaticity condition takes the simple form

$$
|\dot{\theta}| \ll \frac{\kappa_{a b}}{\sin \theta}
$$

or equivalently, substituting $\theta$ and $\dot{\theta}$ from (41)

$$
\left|\frac{\dot{\kappa}_{c b}}{\kappa_{c b}}-\frac{\dot{\kappa}_{a b}}{\kappa_{a b}}\right| \ll\left(\kappa_{a b}^{2 / 3}\left(\frac{\kappa_{a b}}{\kappa_{c b}}\right)^{2 / 3}+\kappa_{c b}^{2 / 3}\left(\frac{\kappa_{c b}}{\kappa_{a b}}\right)^{2 / 3}\right)^{3 / 2} .
$$

It can be observed in the equation above that the adibaticity condition is satisfied if the area of the coupling coefficient is large enough and the relative change with $z$ is smooth enough. As the detuning increases the adiabaticity condition becomes more restrictive and the adiabatic approximation is increasingly less valid.

\section{ACKNOWLEDGMENT}

The authors wish to thank O. Painter for useful discussions on non-Hermitian systems and to A. Bäcker for his encouragement during the realization of this work.

\section{REFERENCES}

[1] A. Yariv, "Coupled mode theory for guided wave optics," IEEE $J$. Quantum Electron., vol. 9, p. 919, 1973.

[2] H. Kogelnik and C. V. Shank, "Coupled wave theory of distributed feedback lasers," J. Appl. Phys., vol. 43, p. 2328, 1972.

[3] G. Meltz, W. W. Morey, and W. H. Glenn, "Formation of Bragg gratings in optical fiber by a transverse holographic method," Opt. Lett., vol. 14, no. 15 , pp. 823-825, 1989.

[4] A. Yariv, Optical Electronics In Modern Communication, 5th ed. New York: Oxford University Press, ch. 13.

[5] E. Kapon, J. Katz, and A. Yariv, "Supermode analysis of phase-locked arrays of semiconductor lasers," Opt. Lett., vol. 9, p. 125, 1984.
[6] R. P. St. J., "Bloch wave analysis of dispersion and pulse propagation in distributed feedback structures," J. Modern Opt., vol. 38, p. 1599, 1991.

[7] E. Peral and J. Capmany, "Generalized Bloch wave analysis for fiber and wave-guide gratings," J. Lightwave Technol., vol. 15, pp. 1295-1302, Aug. 1997.

[8] A. Yariv and P. Yeh, Optical Waves in Crystals. J. Wiley and SonsInterscience, 1984, ch. 6.

[9] A. S. Kewitsch, G. A. Rakuljic, P. A. Willems, and A. Yariv, "All fiber zero-insertion loss add-drop filter for wavelength-division multiplexing," Opt. Lett., vol. 23, p. 206, 1998.

[10] D. Marcuse, Theory of Dielectric Optical Waveguides. New York: Academic, 1974, p. 106.

[11] S. S. Orlov, A. Yariv, and S. Van Essen, "Coupled-mode analysis of fiber optic add-drop filters for dense wavelength-division multiplexing," Opt. Lett., vol. 22, p. 688, 1997.

[12] M. S. Walen et al., "Demonstration of a narrowband Bragg-reflection filter in a single mode fiber directional coupler," Electron. Lett., vol. 22, no. 12 , p. $681,1986$.

[13] M. C. Pease, Method of Matrix Algebra. New York: Academic, 1965.

[14] K. O. Hill, B. Malo, K. A. Vineberg, F. Bilodeau, D. C. Johnson, and I. Skinner, "Efficient mode conversion in telecommunication fiber using externally written gratings," Electron. Lett., vol. 26, no. 16, pp. 1270-1272, Aug. 1990.

[15] M. Abramowitz and I. Stegun, Handbook of Mathematical Functions: With Formulas, Graphs, and Mathematical Tables. New York: Dover, 1976.

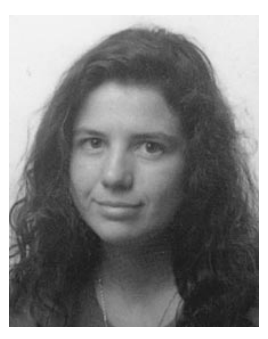

Eva Peral was born in Granada, Spain, on November 23, 1972. She received the degree of Ingeniero Superior de Telecomunicaciones with Honors from the Universidad Politécnica de Valencia (UPV), Spain, in 1995, the M.S. degree in electrical engineering from the California Institute of Technology (Caltech), Pasadena, CA, in 1998, and the Ph.D. degree in telecommunications engineering from UPV in 1998. Since 1996, she has been at Caltech and is currently working towards the Ph.D. degree in electrical engineering.

During 1995, she was a visiting student at the University of Strathclyde, Glasgow, Scotland, U.K., and collaborated with the Optoelectronics Group where she was engaged in integrated optics. She has worked for Philips, Valencia, Spain, and Mercedes-Benz, Sindelfingen, Germany. She started her doctoral studies with the Optical Communications and Photonic Engineering Group in UPV. Her research interests include semiconductor lasers, fiber gratings, linear and nonlinear propagation in optical fiber, and integrated optics.

Ms. Peral received the First National Award for Academic Excellency from the Spanish Ministry of Culture and Education in 1996.

Amnon Yariv (S'56-M'59-F'70-LF'95), photograph and biography not available at the time of publication. 